\title{
Signatures of Electronic Nematic Phase at Isotropic-Nematic Phase Transition
}

\author{
Hae-Young Kee ${ }^{1}$, Eugene H. Kim ${ }^{1,2}$, and Chung-Hou Chung ${ }^{1}$ \\ 1 Department of Physics, University of Toronto, Toronto, Ontario, Canada M5S 1A7 \\ ${ }^{2}$ Department of Physics and Astronomy, McMaster University, Hamilton, Ontario, Canada L8S $4 M 1$
}

(Dated: November 13, 2018)

\begin{abstract}
The electronic nematic phase occurs when the point-group symmetry of the lattice structure is broken, due to electron-electron interactions. We study a model for the nematic phase on a square lattice with emphasis on the phase transition between isotropic and nematic phases within mean field theory. We find the transition to be first order, with dramatic changes in the Fermi surface topology accompanying the transition. Furthermore, we study the conductivity tensor and Hall constant as probes of the nematic phase and its transition. The relevance of our findings to Hall resistivity experiments in the high- $T_{c}$ cuprates is discussed.
\end{abstract}

PACS numbers: 71.10.Hf,72.15.Eb,72.80.Ga

\section{INTRODUCTION}

Recently, novel spatially inhomogeneous and/or anisotropic phases have been found to occur in various condensed matter systems. Similar to classical liquid crystals, [1] these quantum phases can be classified according to their broken symmetries. One such phase is the smectic phase, often referred to as a stripe phase. This phase can be viewed as an unidirectional charge density wave phase which spontaneously breaks translational symmetry along one direction. Experimental evidence for smectic phases has been found in several transitionmetal oxide compounds. In particular, a stripe crystal phase has been observed in manganese oxide compounds by electron diffraction. 2] Moreover, neutron scattering experiments have provided indirect evidence for stripe phases in the high- $T_{c}$ cuprate superconductors. 3 ]

Another such phase is the nematic phase - an anisotropic phase with broken rotational symmetry, but with translational symmetry in tact. This phase can be thought of as a smectic phase "melted" via the proliferation of topological defects. Nematic phases have been argued to be the source of the anisotropic transport observed in quantum Hall systems in high Landau levels [4, [5], and to be relevant to the high- $T_{c}$ cuprate superconductors as well. [6] However, an understanding of the transition from the smectic to nematic phase due to the proliferation of the topological defects still remains to be achieved.

Recently, a complementary approach to studying the nematic phase was suggested. 7, 8] In this approach, the nematic phase is obtained from an isotropic liquid by breaking rotational symmetry. In the continuum limit, it was shown that the Goldstone mode resulting from breaking rotational symmetry leads to non-Fermi liquid behavior in the single-particle lifetime. 7] Exotic superconducting instabilities mediated by the collective mode in the nematic phase were also studied. [9]

While the consequences of the nematic order in the recent complementary approach were studied in Ref. 10, 11, we still lack an understanding of the phase transition between the isotropic and nematic phases. In this work, we study a model for the nematic phase on a square lattice, with emphasis on the phase transitions between the isotopic and nematic phases. Furthermore, we study the conductivity tensor and the Hall constant as probes of nematic phase and its transition.

\section{MODEL HAMILTONIAN AND PHASE TRANSITIONS}

The Hamiltonian we consider is

$$
H=\sum_{\mathbf{k}} \xi_{\mathbf{k}} c_{\mathbf{k}}^{\dagger} c_{\mathbf{k}}+\sum_{\mathbf{q}} F_{2}(\mathbf{q}) \operatorname{Tr}[\hat{Q}(\mathbf{q}) \hat{Q}(-\mathbf{q})]
$$

In Eq. 1 $\xi_{\mathbf{k}}$ is the single-particle dispersion; $F_{2}(\mathbf{q})$ is the inter-electron interaction strength, which can be written as

$$
F_{2}(\mathbf{q})=\frac{F_{2}}{1+\kappa q^{2}}
$$

$\hat{Q}(\mathbf{q})$ is the quadrupole density tensor

$$
\hat{Q}(\mathbf{q})=\frac{1}{\sqrt{N}} \sum_{\mathbf{k}} c_{\mathbf{k}+\frac{\mathbf{q}}{2}}^{\dagger}\left(\begin{array}{cc}
\cos k_{x}-\cos k_{y} & \sin k_{x} \sin k_{y} \\
\sin k_{x} \sin k_{y} & \cos k_{y}-\cos k_{x}
\end{array}\right) c_{\mathbf{k}-\frac{\mathbf{q}}{2}},
$$

where $N$ is the number of lattice sites. In what follows, we take $\xi_{\mathbf{k}}$ to arise from a tight-binding model on a square lattice with nearest-neighbor $(t)$ and next-nearest neighbor $\left(t^{\prime}\right)$ hopping integrals

$$
\xi_{\mathbf{k}}=-2 t\left(\cos k_{x}+\cos k_{y}\right)-4 t^{\prime} \cos k_{x} \cos k_{y}-\mu,
$$

where $\mu$ is the chemical potential, and we have set the lattice spacing to unity, $a=1$.

Within a mean field approximation, we have the selfconsistency equations

$$
\begin{aligned}
\left\langle Q_{x x}\right\rangle & =\frac{1}{N} \sum_{\mathbf{k}}\left(\cos k_{x}-\cos k_{y}\right) f\left(\xi_{\mathbf{k}}\right), \\
\left\langle Q_{x y}\right\rangle & =\frac{1}{N} \sum_{\mathbf{k}}\left(\sin k_{x} \sin k_{y}\right) f\left(\xi_{\mathbf{k}}\right), \\
n & =\frac{1}{N} \sum_{\mathbf{k}} f\left(\xi_{\mathbf{k}}\right),
\end{aligned}
$$




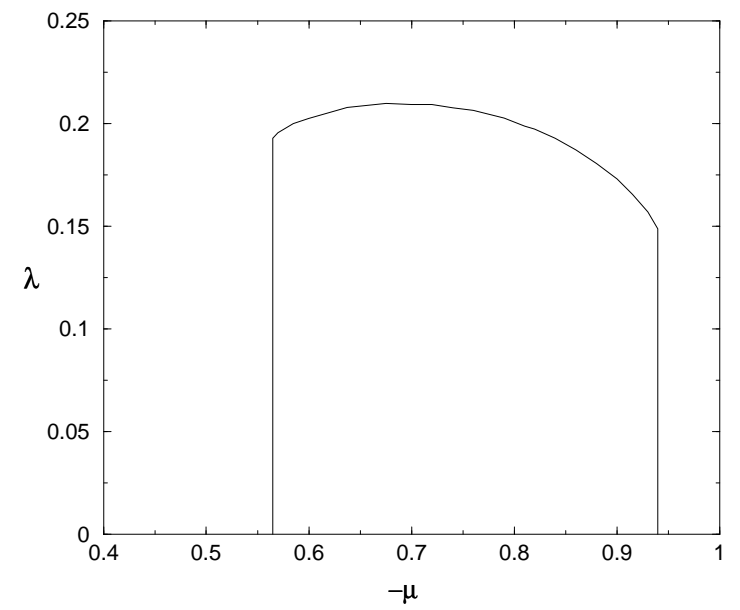

FIG. 1: The nematic order $\lambda$ as a function of chemical potential, $\mu$ for $\left|F_{2}\right| /(2 t)=0.55$ and $t^{\prime}=-0.4 t(2 t \equiv 1)$ at $T=0$. $\mu=-0.4$ corresponds to the half filling, $x=0$

where $f\left(\xi_{\mathbf{k}}\right)$ is the Fermi function, and $n$ is the electron density. The expectation values $\left\langle Q_{x x}\right\rangle$ and $\left\langle Q_{x y}\right\rangle$ lead to a modification of the single-particle dispersion. Defining

$$
\lambda=\left|F_{2}\right|\left\langle Q_{x x}\right\rangle /(2 t) \text { and } \lambda^{\prime}=\left|F_{2}\right|\left\langle Q_{x y}\right\rangle /\left(4 t^{\prime}\right),
$$

the single particle dispersion becomes

$$
\begin{aligned}
\xi_{\mathbf{k}} & =-2 t\left[(1+\lambda) \cos k_{x}+(1-\lambda) \cos k_{y}\right] \\
& -4 t^{\prime}\left(\cos k_{x} \cos k_{y}+\lambda^{\prime} \sin k_{x} \sin k_{y}\right)-\mu .
\end{aligned}
$$

For given values of $\mu, F_{2}$, and $t^{\prime}$, we solved for $\lambda, \lambda^{\prime}$ and the density $n=1-x$ ( $x$ is the hole concentration) using Eq. 2. For all values of the parameters we considered, we found $\lambda^{\prime}=0$.

In Fig. 10 we show the behavior of the nematic order $\lambda$ as a function of chemical potential, $\mu$ for $F_{2} /(2 t)=$ $-0.55, t^{\prime}=-0.4 t$, and $2 t \equiv 1$ at $T=0$. An important feature of Fig. 1 is the sharp onset and offset of nematic order at critical values of $\mu: \mu_{c}=-0.57$ and -0.94 . The free energy near the transition $--0.61 \leq \mu \leq-0.53$ and $-0.96 \leq \mu \leq-0.89-$ shows three minima at finite $\pm \lambda$ and $\lambda=0$. This indicates a region of coexistence of the isotropic and nematic phases: it reflects the negative quartic term in the Ginzburg-Landau free energy. 12 Hence, the transition between the isotropic and nematic phases is first order. The electron density as a function of $\mu$ shows a discontinuity at these critical values of $\mu_{c}$, which is a signature of the first order transition.

To better understand the behavior of the nematic order, as well as the robustness of our result, we considered the dependence of $\lambda$ on the parameters $F_{2}$ and $t^{\prime}$. Upon increasing $\left|F_{2}\right|$, we found the maximum value of $\lambda$ to increase. Also, increasing $\left|F_{2}\right|$ shifts the critical values of $\mu$, making the region over which nematic order exists wider. Furthermore, we found an absence of nematic order for all doping concentrations if $\left|F_{2}\right| /(2 t) \leq 6 \times 10^{-3}$ (within the numerical precision) for $t^{\prime}=-0.4 t$. By varying $t^{\prime}$, we found the structure shown in Fig. 1 to shift.
In particular, when $t^{\prime}=0, \lambda$ is maximal at $\mu=0$ (i.e. half-filling) and is a symmetric function of $\mu$. This is a consequence of the particle-hole symmetry present when $t^{\prime}=0$. Though the critical values of $\mu$ and the maximal value of $\lambda$ depend on the value of $F_{2}$ and $t^{\prime}$, we would like to stress that the qualitative features of the phase transition between the isotropic and nematic liquids are not sensitive to the choice of parameters. In what follows, we will present results for $F_{2} /(2 t)=-0.55$, and $t^{\prime}=-0.4 t(2 t \equiv 1)$. For small hole concentrations, this gives a hole-like Fermi surface centered about $(\pi, \pi)$ (see Inset (a) of Fig. 3). Such a Fermi surface is consistent with angle resolved photoemission spectroscopy [13] and Hall constant measurements [14] in the high- $T_{c}$ cuprates.

\section{CONDUCTIVITY TENSOR AND THE HALL CONSTANT}

Above, we saw that the nematic phase occurs in a finite regime dopings. Furthermore, we found a dramatic change in the Fermi surface topology upon developing nematic order. (See the Inset of Fig. [3) In this section, we study the conductivity tensor to probe the nematic order, as the conductivity tensor, especially the hall conductivity is sensitive to the topology of the Fermi surface.

Within a Boltzmann equation approach, the longitudinal conductivities are (in units where $\hbar=1$ )

$$
\sigma_{i i}=2 e^{2} \frac{1}{N} \sum_{\mathbf{k}}\left(-\frac{\partial f}{\partial \xi_{\mathbf{k}}}\right)\left(v_{i}^{2} \tau_{\mathbf{k}}\right)
$$

where $i=x$ or $y, e$ is the electron's charge, $v_{i}$ is the component of the Fermi velocity along the $i$-axis, and $\tau_{\mathbf{k}}$ is the transport lifetime. In the presence of a magnetic field $\mathbf{H}$ parallel to the $\hat{z}$-axis, the Hall conductivity is 15 , 16.

$$
\sigma_{x y}=2 e^{3} H \frac{1}{N} \sum_{\mathbf{k}}\left(-\frac{\partial f}{\partial \xi_{\mathbf{k}}}\right)\left(v_{y} \tau_{\mathbf{k}}\right)\left(\mathbf{v}_{\mathbf{k}} \times \nabla_{\mathbf{k}}\right)_{\hat{z}}\left(v_{x} \tau_{\mathbf{k}}\right) .
$$

In what follows, we will report results for the case $\tau_{\mathbf{k}} \equiv$ $\tau=$ const. Some discussion regarding the form of $\tau_{\mathbf{k}}$ is given in the following Section.

Using the single-particle dispersion in Eq. 4 with $\lambda$ determined from Eq. 2 we plot the conductivity tensor vs. $\mu$ in Fig. 2 For comparison, the conductivity tensor without nematic order $(\lambda=0)$ is shown as a dashed line. When nematic order develops, the Fermi surface topology changes dramatically. More specifically, the holelike Fermi surface with nematic order becomes an open Fermi surface upon developing nematic order. In the nematic state, the Fermi surface is rather flat along the $\hat{y}$-direction. (See Inset (b) of Fig. [3) Hence, the hole's motion along the $\hat{x}$-direction is enhanced, while it is suppressed along the $\hat{y}$-direction. These changes in the Fermi surface are reflected in the longitudinal conductivities, Figs. 2(a) and 2(b) $-\sigma_{x x}$ increases suddenly with the 

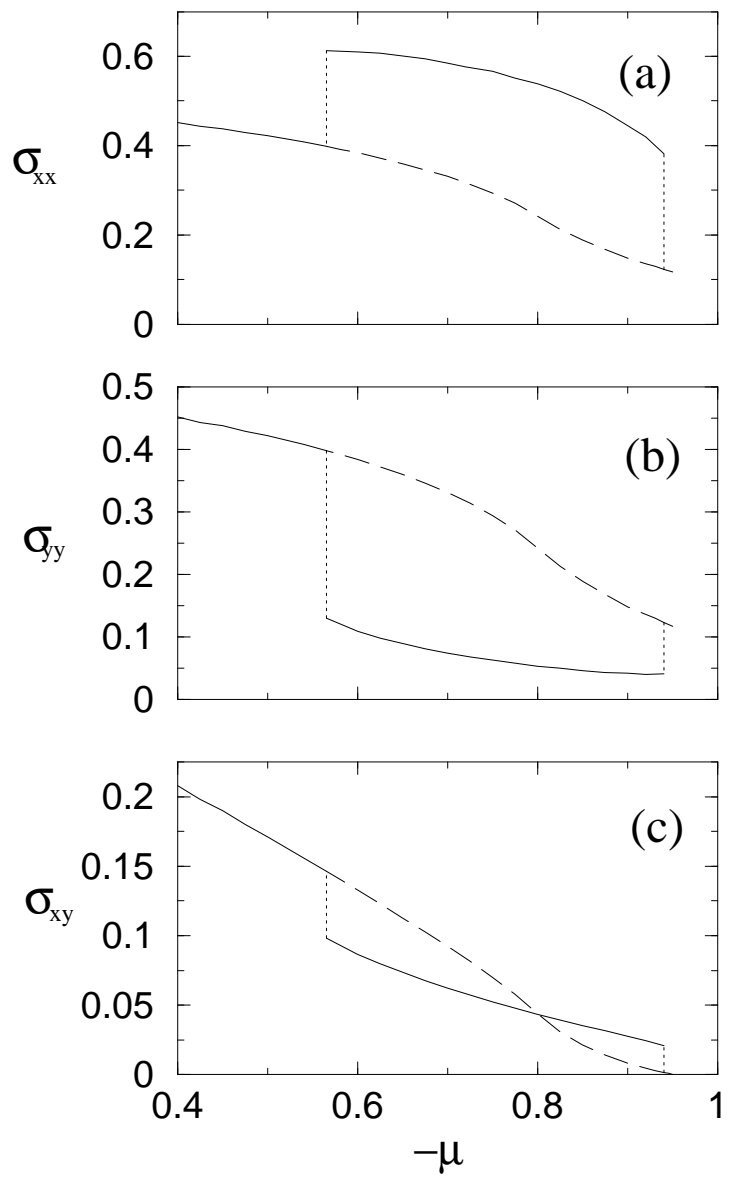

FIG. 2: The conductivity tensor as a function of chemical potential $\mu$ for $\left|F_{2}\right| /(2 t)=0.55$ and $t^{\prime}=-0.4 t(2 t \equiv 1)$. (a) $\sigma_{x x}$ vs. $\mu$, (b) $\sigma_{y y}$ vs. $\mu$, and (c) the Hall conductivity $\sigma_{x y}$ vs. $\mu$. $\sigma_{x x}$ and $\sigma_{y y}$ are in units of $2 e^{2}(2 t)^{2} ; \sigma_{x y}$ is in units of $2 e^{3} H(2 t)^{3}$. The dramatic change is due to the phase transition to nematic state. The dashed lines indicate the behavior of conductivities without the nematic order.

onset of nematic order (at $\mu=-0.57$ ), while $\sigma_{y y}$ shows a sudden decrease. The Hall conductivity, Fig. 2(c), shows a dramatic drop at $\mu=-0.57$ as well. However, its change is small compared with $\sigma_{x x}$ and $\sigma_{y y}$. This occurs because the Hall conductivity is not only sensitive to the Fermi surface's topology, but also its curvature. 15] As shown by the dashed line in Fig. 2(c), the Hall conductivity already decreases fairly rapidly without nematic order. The dotted lines at the transition indicate that Boltzmann equation, Eq. 5 is not applicable near the transition, since there are two phases with different densities coexist.

Another quantity of interest is the Hall constant $n_{H}$, which measures the number of charge carriers, and is related to the Hall resistivity. For weak magnetic fields, the Hall resistivity is

$$
\rho_{x y} \simeq \frac{\sigma_{x y}}{\sigma_{x x} \sigma_{y y}}=\frac{1}{n_{H} e c} H,
$$

which defines $n_{H}$ in terms of $\rho_{x y}$. For the case of a single-

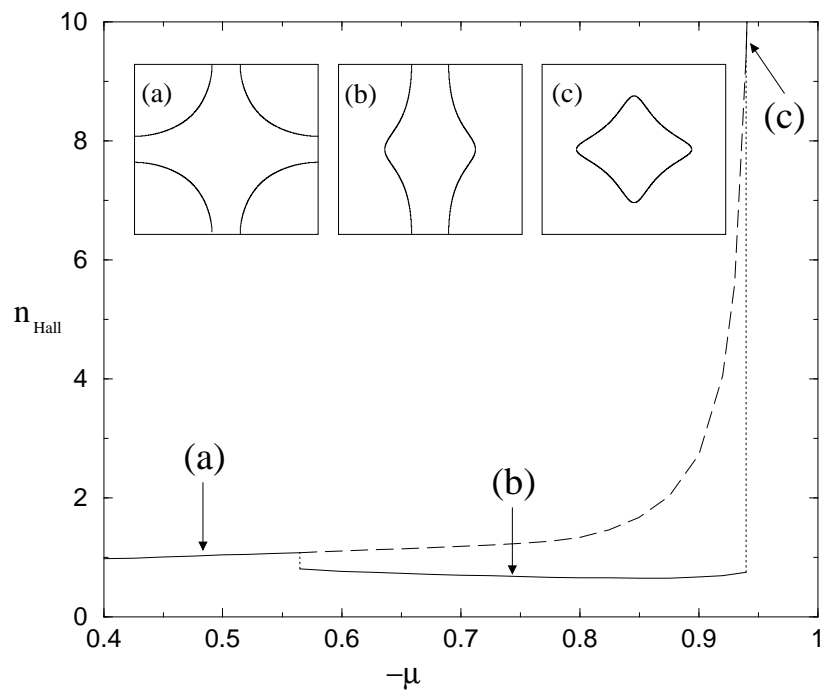

FIG. 3: Hall constant as a function of chemical potential, $\mu$ for $F_{2} /(2 t)=-0.55$ and $t^{\prime}=-0.4 t(2 t \equiv 1)$. The dashed line is the Hall constant without the nematic order. The inset shows the the Fermi surface for the points labeled by arrows in the main figure.

band Fermi liquid model with a circular (or elliptical) Fermi surface, $n_{H}$ is the density of charge carriers per unit cell i.e. the area of the Fermi surface.

In Fig. 3, we plot the Hall constant, $n_{H}$ as a function of chemical potential, $\mu$. For comparison, the Hall number without nematic order $(\lambda=0)$ is shown as a dashed line. Notice the sharp drop of $n_{H}$ at $\mu=-0.57$, where nematic order becomes finite. It is expected that $n_{H}$ is almost constant in the region where nematic order exists, $-0.94 \leq \mu \leq-0.57$. This can be understood from the conductivity tensor in Fig. 2 - the decrease of hole motion along the $y$ direction is compensated by the increase of hole motion along $x$ direction, giving a product $\sigma_{x x} \sigma_{y y} / \sigma_{x y} \sim n_{H}$ which is nearly constant.

\section{DISCUSSION}

To begin with, our results for the phase transition in Fig. [1 were obtained within a mean-field approximation. Once fluctuations about the mean-field state are included, we expect the range over which the nematic phase exists to be reduced. Furthermore, fluctuations will reduce the value of $\lambda$. The effect of fluctuations on the nature of phase transition will be addressed in near future. Another approximation in our treatment was the neglect of collective modes. In the continuum limit of Eq. 11 the breaking of rotational symmetry leads to a Goldstone mode; scattering from this Goldstone mode gives rise to non-Fermi liquid behavior. [7] On a lattice, however, this collective mode is gapped; we expect scattering from the collective mode to have little effect on the $d c$ conductivities.

The subject of disorder deserves some discussion. 
Though disorder was introduced to understand the behavior of the conductivity through the transitions, we did not address its effects on the transition between the isotropic and nematic phases, nor did we address its consequences on the nematic phase itself. 17] In the absence of disorder, the mean-field treatment of the Hamiltonian Eq. 1 gave the transition between the isotropic and nematic phases to be first order. For systems that undergo a first order transition without disorder, it was shown that the system with quenched disorder could be mapped onto a random field Ising model.[18] Based on this mapping, it was shown that in $d \leq 2$ dimensions, the coexistence at the phase transition disappears for arbitrarily small amount of disorder; in $d>2$ dimensions this occurs by the application of a finite amount of disorder. For the transition in this work, however, there are gapless fermions presents on both sides of the transition. Therefore it is unclear to us how much the results obtained from the mapping may apply. We hope to address these issues in the future.

It is also important to note that the conductivity tensor depends of the form of the transport scattering rate, and hence on the disorder. Realistic models for disorder usually give transport scattering rates which are momentum dependent. In particular, in most high- $T_{c}$ materials, disorder is mainly due to the dopant ions, which lie outside of the $\mathrm{CuO}_{2}$ planes. As a result, these dopant ions cause primarily forward scattering. In the above discussion, we described our results for the case $\tau_{\mathbf{k}} \equiv \tau$. However, we have also considered the case where $\tau_{\mathbf{k}} \sim\left|\mathbf{v}_{\mathbf{k}}\right|$. [12] Such a form arises for out-of-plane disorder, causing primarily forward scattering. 19] When $\lambda=0$, we found the overall features of $n_{H}$ to be very different from $\tau_{\mathbf{k}}=\tau$. However, the effect of the nematic order on the Hall constant gives the same result as when $\tau_{\mathbf{k}} \equiv \tau$ - there is a sharp drop in the Hall constant in the nematic phase compared with the isotropic phase.

Our findings for the Hall constant have potential relevance to the experiments of the normal state Hall resistivity in the high- $T_{c}$ superconductor, $\mathrm{Bi}_{2} \mathrm{Sr}_{2-x} \mathrm{La}_{x} \mathrm{CuO}_{6+\delta} \cdot$ 20, 21] In these experiments, the Hall constant was measured in the normal state at low temperatures, by suppressing superconductivity with a large pulsed magnetic field of $60 \mathrm{~T}$. They observed saturation of the Hall constant at low temperatures, suggesting conventional transport properties. However, the Hall constant as a function of doping concentration shows a maximum at $x=0.15$, and decreased sharply in underdoped regime. In overdoped regions, there is a regime near optimal doping $x \simeq 0.16$, where the Hall constant shows the dip - the decrease is about $20 \%(\simeq 0.2)$ of its maximum value $(\simeq 1.0)$ at $x=0.15$. The dip of the Hall constant around $x=0.16$ becomes more pronounced at low temperatures $(<1 K)$. This suggests that the nematic phase may be a candidate to explain the anomaly observed in the Hall constant in high $T_{c}$ cuprates.

In conclusion, to study the nematic phase we considered the Hamiltonian in Eq. 1] with quadrupolar densitydensity interactions on a square lattice. Within a meanfield analysis, we found the nematic phase to occur in a finite region of dopings. Moreover, the transition between the isotropic and nematic phases was found to be first order; a dramatic change in the Fermi surface topology accompanied this transition. As the conductivity is sensitive to the topology of the Fermi surface, we investigated the effects of nematic order on transport. We found the conductivity tensor and Hall constant to show significant changes at the transitions between the isotropic and nematic phases. If observed, such signatures would give evidence for nematic order; it would give evidence for the approach pursued in this paper as an appropriate description of nematic phases in physical systems. Finally, we discussed the effect of disorder and the scattering from collective modes on the phase transitions and on the transport properties at the transitions.

Acknowledgments We would like to thank S. A. Kivelson, V. Oganesyan P. Coleman, Y-B Kim, and E. Fradkin for insightful discussions, and Yoichi Ando for useful comments. We also thank P. Fournier and W. Metzner for pointing out Ref. 14 and Ref. 8, respectively. This work was supported by the NSERC of Canada(HYK,EHK,CHC), Canada Research Chair(HYK), Canadian Institute of Advanced Research(HYK), Alfred P. Sloan Research Fellowship (HYK), and Emerging Material Knowledge program funded by Materials and Manufacturing Ontario (HYK).
[1] Physics of Liquid Crystals, P. de Gennes ( Oxford University Press, 1987).

[2] S. Mori, C. H. Chen, and S. W. Cheong, Nature, 392, 473 (1998).

[3] J. Tranquada, B. J. Sternlieb, J. D. Axe, Y. Nakamura, and S. Uchida, Nature, 375, 561 (1995).

[4] M. P. Lilly, K. B. Cooper, J. P. Eisenstein, L. N. Pfeiffer, and K. W. West, Phys. Rev. Lett. 82, 394 (1999); R. R. Du, D. C. Tsui, H. L. Stormer, L. N. Pfeiffer, K. W. Baldwin, K. W. West, Solid State Comm. 109, 389 (1999); K. B. Cooper, M.P. Lilly, J. P. Eisenstein, L. N. Pfeiffer, K. W. West, Phys. Rev. B 65, 241313 (2002).
[5] E. Fradkin and S. A. Kivelson, Phys. Rev. B 59, 8065 (1999).

[6] S. A. Kivelson, E. Fradkin, and V. J. Emery, Nature 393, 550 (1998).

[7] V. Oganesyan. S. Kivelson, and E. Fradkin, Phys. Rev. B 64, 195109 (2001).

[8] C. J. Halboth, and W. Metzner, Phys. Rev. Lett. 85, 5162 (2000); A. Neumayr, and W. Metzner, Phys. Rev. B 67, 035112 (2003); V. Hankevych, and F. Wegner, Eur. Phys. J. B 31, 333 (2003).

[9] Y. B. Kim and H.-Y. Kee, cond-mat/0204037

[10] H.-Y. Kee, Phys. Rev. B, 67, 073105 (2003). 
[11] S. A. Kivelson, E. Fradkin, V. Oganesyan, I. P. Bindloss, J. M. Tranquada, A. Kapitulnik, and C. Howald, cond-mat/0210683 to be published in Rev. Mod. Phys.

[12] C. H. Chung, H.-Y. Kee, and E. H. Kim, unpublished.

[13] See the review article: J. C. Campuzano, M. R. Norman, and M. Randeria, in in Physics of Conventional and Unconventional Superconductors, edited by K. H. Bennemann and J. B. Ketterson (Springer-Verlag).

[14] H. Y. Hwang, B. Batlogg, H. Takagi, H. L. Kao, J. Kwo, R. J. Cava, J. J. Krajewski, and W. F. Peck, Jr., Phys. Rev. Lett. 72, 2636 (1994).

[15] N. P. Ong, Phys. Rev. B 43, 193 (1991).

[16] J. Ziman, Electrons and Phonons (Clarendon, Oxford 1960).

[17] The effect of the disorder on electronic liquid crystal is one of the interesting issues. For the classical liquid crystal in the continuum, see L. Radzihovsky and J. Toner, Phys. Rev. B 60, 206 (1999).

[18] J. Cardy, cond-mat/9806355 See the references therein.

[19] H.-Y. Kee, Phys. Rev. B 64, 012506 (2001).

[20] F. F. Balakire, J. B. Betts, S. Ono, T. Murayama, Y. Ando, and G. S. Boebinger, in Physical Phenomena at High Magnetic Fields-IV edited by G. Boebinger, Z. Fisk, L. P. Gorkov, A. Lacerda, and J. R. Schrieffer (world Scientific, Singapore, 2002).

[21] A theoretical interpretation was given in S. Chakravarty, C. Nayak, S. Tewari, and X. Yang, Phys. Rev. Lett. 89, 277003 (2002). 